\title{
Soviets' pair of Mars probes paving the way for man
}

\section{London \& Washington}

THE Soviet Union's most ambitious planetary mission ever got off to a successful start last Thursday with the launch of Phobus-1, the first of two 6-tonne spacecraft that will rendezvous next year with Mars. The launch of Phobus-2 was scheduled for Tuesday this week.

When they reach Mars, the spacecraft will survey the planet from orbit for 120 days, taking photographs, recording infrared and gamma-ray spectrograms, and collecting data on the solar wind and solar radiation.

Phobus-2 will then transfer to an orbit about the martian moon Phobus and carry out similar observations. Finally, next May it will make a 20 -minute flyby of the satellite, approaching to within 50 metres of the surface, moving with a relative velocity of 2 to 5 metres a second - almost walking pace.

During the flyby Phobus-2 will use a laser to ionize soil on Phobos, while a mass spectrometer will analyse the resulting vapour. A radar beam will also take soundings of the moon for surface structure analysis.

After completing the survey, Phobus-2 will drop a "long-life autonomous station" to the surface of Phobus. This has been assigned a 3-month programme using Xray and alpha backscatter spectrometry to study surface composition. Cameras will take close-up pictures and a seismometer will measure seismic activity.

Phobus-2 will also drop a mobile lander to the surface of Phobos. Dubbed the hopper, this lander will make 20-metre leaps on spring-loaded legs, giving a wider distribution of sample sites.

The United States and Soviet Union have been recently making friendly overtures towards each other over Mars. Each country will have scientific teams participating in the other's Mars missions. The next US mission is the Mars Observer, scheduled for launch in 1992.

The National Aeronautics and Space Administration (NASA) is not providing any hardware for the Phobos mission, but the US space agency does have a significant part to play in helping the Phobos spacecraft arrive at the right place at the right time.

Using very-long baseline interferometry (VLBI), NASA's deep space network will pinpoint the location of the Phobos spacecraft starting this autumn. This technique, now standard for tracking spacecraft, uses two of the three deep space network antennas (one in southern California, one in Spain, and one in Canberra, Australia) to record signals from the spacecraft simultaneously to determine its angular position relative to a reference frame provided by quasar radio sources.

In April, communications hardware from Phobos was tested at the Goldstone (California) tracking site to assure that the US and Soviet equipment was compatible. NASA will continue to use the radio beacon on the Phobos landers to conduct VLBI studies, but there are two other instruments on the landers that NASA scientists will have access to. One will be used to study Phobos' wobble - libration - during its 7 hour and 39 minute-rotational period. The rotational period is the same as its orbital period, so one face is constantly pointed towards Mars.

But slight vibrations that will be detected by measuring the angular position of the Sun from Mars will be used to deduce the internal distribution of mass of Phobos. This should help determine whether Phobos is a captured asteroid.

A second instrument will be used for ranging studies. A relayed signal transmitted from Earth will allow precise determination of Phobos' distance to an accuracy of 10 metres.

Robert Preston of JPL, who serves as NASA's chief scientist for Phobos activities, says Phobos is rapidly - in celestial terms - spiralling in towards Mars, and in another 50 million years may crash into the planet or break apart under tidal forces. Precise knowledge of Phobos's orbit should also give a better idea of the Martian gravitational field, and may provide information about the mass of nearby asteroids.

An even more exciting experiment, according to Preston, will be a more precise determination of the gravitational constant. Preston says the the Solar System provides a marvellous laboratory for conducting experiments on general relativity. It may even be possible to detect changes in the gravitational constant by comparing Phobos data with similar data collected by the US Viking landers in the 1970s.

Soviet commentators are placing great emphasis on two aspects of the flight. One is the extensive international participation, Nineteen nations are involved, either as suppliers of experiments and hardware or as trackers including the United States, the United Kingdom, Australia, Sweden, Finland, Ireland, West Germany and Yugoslavia, as well as the Comecon block. The other is the idea that this is another step towards a manned mission to Mars. During the past three years, recordbreaking stays aboard the Salyut and Mir orbital stations have been officially described as the necessary first step to Mars. Joseph Palca \& Vera Rich
NASA criticized on safety

\section{Washington}

WITH the space shuttle Discovery on the pad, ready for launching in September, NASA's commitment to the maintenance of a strong safety management and risk assessment programme is being questioned. In a report published last week, an ad hoc committee chaired by Charles Mertz of NASA headquarters and Joyce McDevitt, a private consultant, warned against a lapse into the attitudes of "business as usual" once Discovery is safely in orbit.

The committee, asked by NASA to judge how well it has dealt with criticism of its previous attention to safety matters, says that for the moment internal discussion of risks and their remedies is unfettered and that morale is high. It found no fault in plans for Discovery's impending launch. But the committee is plainly unsure that the present commitment to making safety the overriding con-

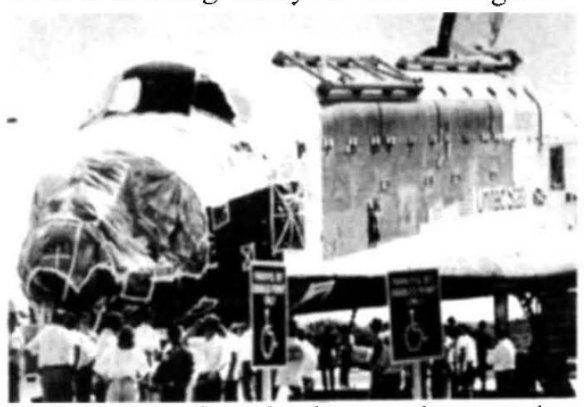

The space shuttle Columbia, on the move last week into the Orbital Processing Facility vacated by Discovery. The date for Discovery's next launch has now been put back to the first week in September. Meanwhile, Columbia - seen above with some parts missing and with plastic covers to protect it from the elements - will be refurbished with the latest safety improvements.

cern in the resumption of shuttle flights will continue past the first re-launch.

Acknowledging that in the two and a half years since the Challenger disaster, many senior managers have been devoted full-time to safety issues, and that the procedures for risk control have been completely rewritten, the report warns that when the need to keep to a regular schedule reasserts itself, people will return to their usual duties and there will be little time to review and alter procedures.

To guard against laxity, the committee argues that more permanent staff specializing in safety questions are needed, and that personnel training and assessment should formally cover safety management. Because the driving force behind recent efforts has been largely individual enthusiasm motivated by public concern, the report says there is a danger that if institutional cnanges are not made now, the present commitment to safety could evaporate.

David Lindley 